\title{
Mechanical Prophylaxis for Post-Traumatic VTE: Stockings and Pumps
}

\author{
Jason Weinberger ${ }^{1} \cdot$ Mark Cipolle $^{1,2}$
}

Published online: 26 February 2016

(C) Springer International Publishing AG 2016

\begin{abstract}
Trauma patients pose unique challenges when it comes to the decision for optimal thromboprophylaxis. Risk reduction of venous thromboembolic (VTE) events is paramount in the modern management of trauma patients. The choice of pharmacologic prophylaxis is standard for most
\end{abstract}

\section{Key Points}

- DVT rates in trauma patients can be as high as $58 \%$ without VTE prevention.

- Mechanical compression of veins facilitates venous emptying by altering pressure gradients, decreases stasis, and provides endogenous fibrinolytic activity.

- Intermittent pneumatic compression devices are favored over graduated compression stockings due to improved efficacy and better patient compliance.

- The application of intermittent pneumatic compression devices in addition to graduated compression stockings offers no additive benefit with regard to VTE outcomes.

- No statistical difference is seen in VTE outcomes with comparison of thigh-length compression versus knee length.

- Upper-extremity pneumatic compression devices may improve endogenous fibrinolytic activity, but studies of clinical VTE outcomes are still sparse.

- For patients with contraindications to pharmacologic prophylaxis, mechanical prophylaxis is recommended via lower extremity compression. - Mechanical compression alone reduces the risk of DVT by 50-60\%.

This article is part of the Topical Collection on Venous Thromboembolism After Trauma

Mark Cipolle

MCipolle@christianacare.org

Jason Weinberger

JWeinberger@christianacare.org

1 JHA Education Center, Christiana Care Health Systems, 4755 Ogletown-Stanton Road, Suite 2E70-B, Newark, DE 19718, USA

2 Department of Trauma, Kidney Kimmel School of Medicine, 4755 Ogletown-Stanton Road, Suite 1320, Newark, DE 19718, USA hospitalized patients. However, the potential for bleeding complications with this therapy, in a subset of major trauma patients, can have dire consequences. Fortunately today, a variety of mechanical compression methods exist to assist in the optimal management of VTE prophylaxis for trauma patients. Graduated compression stockings, pneumatic compression devices, foot pumps, and neuromuscular electrical stimulators are just a few of the choices in today's providers' armamentarium. In this review, we discuss the need for mechanical VTE prophylaxis in trauma patients, the mechanisms of clot prevention associated with the available devices, and take an evidence-based look at the efficacy of the mechanical methods available for VTE prophylaxis.

Keywords Mechanical prophylaxis · Trauma venous thromboembolism (VTE) prophylaxis · Pneumatic compression devices · Compression stockings - Venous foot pumps

\section{Introduction}

Much has been written about the prevention of venous thromboembolism (VTE) since Rudolf Virchow first described the phenomenon in 1846 [1]. His often quoted triad of stasis, endothelial damage, and hypercoagulable state (Fig. 1) are no more prevalent than in the trauma patient population. This link between blood flow and incidence of VTE may be more than a century old, but the innovations in prevention of these events over the last few years have been remarkable.

Trauma and critically ill patients are exceedingly susceptible to VTE events as they are often prone to stasis by their immobility, have sustained endothelial injury from their trauma, and find their own immune system up regulated to a hypercoagulable state [2]. In a landmark study of trauma 


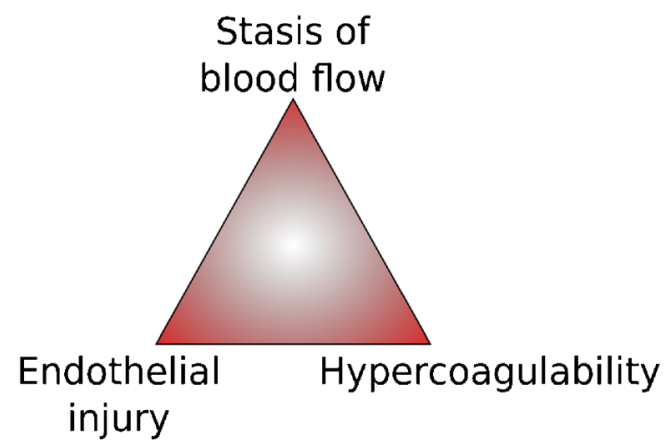

Fig. 1 Virchow's triad of thrombosis. Stasis of blood flow, endothelial injury, and hypercoagulability are three factors that contribute to the pathogenesis of venous thrombosis. Trauma patients not only are exposed to these risk factors but often possess all three concomitantly [1]

patients not receiving chemoprophylaxis, Geerts et al. reported deep vein thrombosis (DVT) rates as high as $58 \%$, and a fatal pulmonary embolism (PE) rate of $0.4 \%$ [3]. The current gold standard for prevention of VTE events in major trauma patients is early pharmacologic prophylaxis with the use of low dose unfractionated heparin (LDUH) or low molecular weight heparin (LMWH). For patients with contraindications to pharmacologic prophylaxis, and who are at risk for VTE, mechanical prophylaxis is recommended via lower-extremity compression [4•]. The role of mechanical devices for prevention of VTE events is in increasing demand as pharmacologic prophylaxis is often, at least, temporarily contraindicated in trauma or critically ill patients for procedures or clinical conditions. Other unique contraindications are seen in trauma patients whose injuries prohibit placement of these mechanical prophylaxis devices due to large dressings or splints. With heightened attention to VTE prophylaxis, and proper application of mechanical prophylaxis devices, VTE events in the hospitalized patient can be reduced [5•].

It is worth emphasizing that all modalities used in the evidence-based approach of VTE prevention should always be interpreted in the complex context of individualized patient care and not simply a comprehensive meta-analysis. No two patients and/or their injury patterns are created equally, and therefore we, as the provider, must at times customize our approach to our patient's VTE risk reduction individually.

\section{Pathophysiology}

Mechanical prophylaxis for VTE prevention comes in a variety of forms, but all are generally small variations on the same physiologic principle: increasing blood velocity decreases venous stasis and thrombosis. Different forms of mechanical prophylaxis alter properties in various ways such as peak venous velocity, flow rate augmentation, and duration of effect. This variance between methods of prophylaxis has been the source of debate with regard to optimal prophylaxis.
During normal activities of daily living, venous return from the legs is propelled by contraction of the calf muscles that then help enhance blood return to the heart. For immobile trauma patients, however, stasis can allow the venous blood to pool in the intramuscular sinuses of the leg, which have become dilated during prolonged rest.

Stockings exert external circumferential pressure on regions of the leg to decrease the diameter of these venous vessels. As Poiseuille's law and venous hemodynamics dictate, this reduction in diameter increases venous blood velocity and promotes venous return. The theory of limited venous distension and subsequent micro-endothelial trauma caused by the dilation of these vessels is also aided by the application of stockings. An additional benefit of circumferential compression is the increase in hydrostatic forces on the extracellular interstitial spaces, which may augment flow and decrease edema.

Pneumatic compression devices have multiple mechanical effects in the prevention of VTE events. When compression is applied (uniformly or sequentially) the change in pressure gradient in the zone of compression accelerates blood forward and facilitates venous emptying while decreasing stasis. This increase in the arterial-venous pressure gradient also enables an increase in arterial blood flow and decreases peripheral vascular resistance [6].

Beyond simply overcoming stasis, some forms of mechanical VTE prophylaxis also appear to offer fibrinolytic properties to combat the hypercoagulable component of Virchow's triad. The increased venous velocity through vessels caused by pneumatic compression devices also triggers endogenous fibrinolytic activity. Studies have revealed a reduction in plasminogen activator inhibitor-1 (PAI-1) and a net increase in tissue plasminogen activator (tPA) activity in participants wearing these devices [7].

\section{Graduated Compression Stockings}

Initially designed for management of chronic conditions such as venous leg ulcers and lymphedema, graduated compression stockings have found an additional niche in the prevention of VTE events. The graded circumferential pressure exerted from distal to proximal regions of the leg by these stockings decreases the diameter of venous vessels, improves venous flow velocity, avoids venous stasis, and overall, improves venous return from the extremities to which they are applied. These effects of the compression stockings are undoubtedly aided by muscular activity of the limb and may have less efficacy in an immobile patient.

The pressure gradient exerted by these stockings decreases from distal to proximal on the extremity. The degree of external pressure exerted by these stockings can lead to therapeutic compression or a tourniquet effect depending on the variation of tension applied by the elastic garments. A typical stocking 
profile of $18 \mathrm{~mm} \mathrm{Hg}$ at the ankle, $14 \mathrm{~mm} \mathrm{Hg}$ at the calf, and $8 \mathrm{~mm} \mathrm{Hg}$ at the thigh improves blood flow velocity with the aid of external compression and increases venous return by $75 \%$ [8]. More does not always equate with better - as Kakker proved in a physiologic assessment that pressure ranges greater than $20 \mathrm{mmHg}$ produced an increased venous velocity, but at the cost of significant impairment in the subcutaneous tissue blood flow. [9]. Due to this risk of a tourniquet effect, it is recommended to check for formation of pressure sores at least every 8 hours during the application of compression stockings.

The efficacy of graduated compression stockings was seen in a systematic review of nineteen randomized control trials of hospitalized patients. Nine of these studies included patients undergoing general surgery; six included patients undergoing orthopedic surgery; and one trial included medical patients. A significant reduction in DVT rates was seen in the treatment group of graduated compression stockings (9\%) in comparison to the control group with no prophylaxis (21\%). An odds ratio (OR) of 0.33 (95\% confidence interval [CI] 0.26-0.41) favored treatment with graduated compression stockings $(p<0.00001)$. A similar odds ratio of 0.38 (95\% CI 0.15 $0.96, p=0.04$ ) was seen in the incidence of pulmonary embolism based on results from five studies that included general or orthopedic surgery patients. The incidence of pulmonary embolism was $2 \%$ in the graduated compression stockings group and $5 \%$ in the no prophylaxis group [10•]. In lieu of anatomic contraindications to the Glasgow Coma Score (GCS) on a lower extremity of a trauma patient, there appears to be benefit of mechanical prophylaxis on the other extremity as seen in a study evaluating GCS on a single extremity versus two. The incidence of DVT in the lower extremity with application of GCS was lower compared to the extremity without mechanical prophylaxis ( 0 versus $10 \%$ ) [11]. Therefore, within the confines of a controlled trial, it appears that graduated compression stockings reduce DVT rates by approximately two thirds when compared to controls.

The lengths of these compression stockings are variable and range from knee length to proximal thigh. A systematic review performed in 2012 evaluated three randomized controlled studies with a combined total of 496 patients to evaluate the role of knee length versus thigh length in thromboprophylaxis among groups of post-operative patients. These studies showed no significant difference in the ability of the two modalities of leg compression to reduce the incidence of deep vein thrombosis in post-operative patients. In both, the fixed-effect model (OR 1.55, $95 \%$ CI $0.78-3.07, p=0.21$ ) and random-effects model (OR 1.32, $95 \%$ CI 0.43-4.06, $p=0.63$ ) [12]. In the absence of evidence to support benefit of thigh length over knee length graduated compression, the use of knee length garments in clinical practice is driven by factors such as ease of application, lower cost, and increased compliance by patients.
The most important impediment for the use of graduated compression stockings has always been compliance by patients. A poor fit and excessive compression can cause complications such as skin marking, blistering, ulceration, pain, discomfort, peroneal palsy, and even case reports of limb ischemia have been cited with the use of graduated compression stockings [13]. Manufacturers offer a variety of pressure profiles and fits, but it still often remains a struggle to find the right garment for the right patient. Newer technologies such as creation of engineered compression stockings using 3 days scanning to create unique garments fit to individual leg profiles with customizable pressure gradients may show promise [14]. This made-to-measure, or "scan to knit", compression garment may not be cost-effective for every patient but perhaps a consideration in the care of a spinal cord injury patient with need for long-term VTE prevention.

\section{Pneumatic Compression Devices}

Historically used for the treatment of chronic lower extremity venous disease and lymphedema, pneumatic compression devices have recently become a mainstay in the prevention of VTE events. The therapeutic benefits of intermittent pneumatic compression devices are both mechanical and biochemical as mentioned earlier, but their true clinical benefit is improved tolerability and efficacy in comparison to graduated compression stockings and avoidance of bleeding complications when compared to pharmacologic prophylaxis.

Efficacy of intermittent pneumatic compression (IPC) devices for prevention for VTE events was assessed in a 2013 meta-analysis of 70 trials including data on 16,164 hospitalized patients (eight trials specifically studied trauma or critically ill patients). Their findings reported that IPC was more effective than no prophylaxis in reducing DVT (7.3 versus. $16.7 \%$, relative risk [RR] $0.43 ; 95 \%$ CI $0.36-0.52$ ) and PE rates ( 1.2 versus $2.8 \%$, RR $0.48 ; 95 \%$ CI $0.33-0.69$ ), without any effect seen on mortality. Further sub-group analysis of nine trials that directly compared IPC devices and GCS alone found IPC devices to be associated with a reduced risk of DVT (RR, 0.61; $95 \%$ CI 0.39-0.93; $p=0.02$,) but not PE (RR, $0.64 ; 95 \%$ CI $0.21-1.95 ; p=0.4$ ). A significant heterogeneity in the risk of DVT among trials existed; however, IPC devices appeared to be as effective as pharmacological thromboprophylaxis in reducing DVT (RR, 0.93; $95 \% \mathrm{CI}$ $0.69-1.26 ; p=0.66)$ and $\mathrm{PE}$ rates (RR, $1.19 ; 95 \% \mathrm{CI} 0.62$ $2.29 ; p=0.59)$. There was no significant difference in mortality seen between the two groups (RR $0.92 ; 95 \% \mathrm{CI}$ $0.44-1.90 ; p=0.81$ ) [5].

The bleeding risk is an important consideration when utilizing pharmacologic prophylaxis in trauma. These patients are particularly challenging since they often have both high bleeding and high clot risk concomitantly. Injured patients are 
predisposed to risk factors from their initial injury, acquired coagulopathies, and recent or upcoming surgical procedures that may incur significant blood loss. A meta-analysis of 3887 trauma patients in 16 studies was performed using a randomeffects model. Without any significant increase in DVT or PE rates, mechanical prophylaxis with compression (GCS or IPC devices) was associated with a significantly reduced risk of post-operative bleeding compared with the heterogenous use of UFH or LMWH (RR 0.47; $95 \%$ CI 0.31-0.70) [15]. The strength of mechanical prophylaxis retains its successful VTE risk reduction in the face of anticoagulation contraindications or significant bleeding risk.

There is a wide variety of IPC devices available (Fig. 2). Similar to the discussion GCS, the length of the garment has long been a subject of debate. The difficulty is that direct comparisons of thigh versus knee IPC devices with regard to VTE outcomes are sparse and often methodologically flawed. In a prospective, randomized, clinical control trial performed by Fedullo et al., the DVT rate of trauma patients receiving thigh length IPC prophylaxis was $2.0 \%$ in comparison to patients receiving calf length IPC prophylaxis who had a DVT rate of $3.9 \%(p=1.00)$ [16]. More patients in the thigh length prophylaxis group, however, were deemed high risk and received concomitant pharmacologic prophylaxis. In another clinical comparison trial of five different pneumatic devices with varying lengths and compression types, the incidence of DVT was found to be $3.6 \%$ in 853 hospitalized patients with calf length IPC sleeves and $3.4 \%$ in 497 hospitalized patients with thigh length IPC sleeves [17]. A recent Cochrane analysis of thromboprophylaxis in trauma patients performed in 2013 reviewed three studies that included 373 patients and found no significant difference in DVT (RR 0.69; $95 \%$ CI $0.37-1.32$ ), PE (RR 0.79; $95 \%$ CI $0.12-5.10$ ) or mortality rates (RR 0.0 , no events reported) in those receiving thigh length mechanical prophylaxis versus below-the-knee devices [18••]. While, in theory, it seems as if compressing more tissue would be more effective in VTE reduction, the data to date dispels that myth.

Another distinguishing variant between IPC devices is their ability to offer sequential or uniform compression. Uniformed compression devices typically exert a single force of $40 \mathrm{mmHg}$ to the calf or thigh level at which they are applied. Active compression normally lasts about $10 \mathrm{~s}$, and the cuff is then allowed to relax for a minute before repeating the cycle. Sequential compression devices have multiple cuffs that inflate distally first and then work more proximal with lower inflation pressures to create a "stripping" action during compression. A study examining the hemodynamics of uniform and sequential compression demonstrated uniform compression resulted in higher peak velocities but sequential compression resulted in a longer augmentation of flow and higher total volume of blood expelled [19]. With the ultimate goal being prevention of VTE events, there has been no proven benefit of one system over another [20]. An additional design variant of pneumatic compression devices is circumferential versus asymmetric compression cuffs. Future comparative human studies are needed to determine a true cost versus benefit of these complex sequential or asymmetric pneumatic compression systems.

Trauma patients with extensive lower extremity injuries pose a conundrum as the application of mechanical prophylaxis devices to these extremities can be limited by massive soft tissue disruption, orthopedic stabilization, and/or amputation. Application of intermittent compression devices on the upper extremities may be of benefit in the prevention of VTE based upon the work of Knight et al. in 1976 as they showed a reduction in lower extremity DVT rates with upper extremity compression devices [21]. The potential for fibrinolytic benefit in prevention of VTE with application of upper extremity compression devices will likely be overshadowed by increased complexity of nursing care, interference with blood pressure monitoring, pulse oximetry tracings, and infusions via peripheral venous catheters.

\section{Venous Foot Pumps}

An alternative to calf compression with graduated compressive stockings or intermittent pneumatic compression devices is the use of foot pumps. The muscles in the foot are much less compressible and the overall blood volume in the plantar venous plexus is much smaller in comparison to the calf, which inevitably requires larger compressive forces to obtain similar
Fig. 2 Pneumatic compressive devices are available in multiple varieties. Pictured are examples of a thigh-length device (a) and a knee-length device (b) with uniform compression
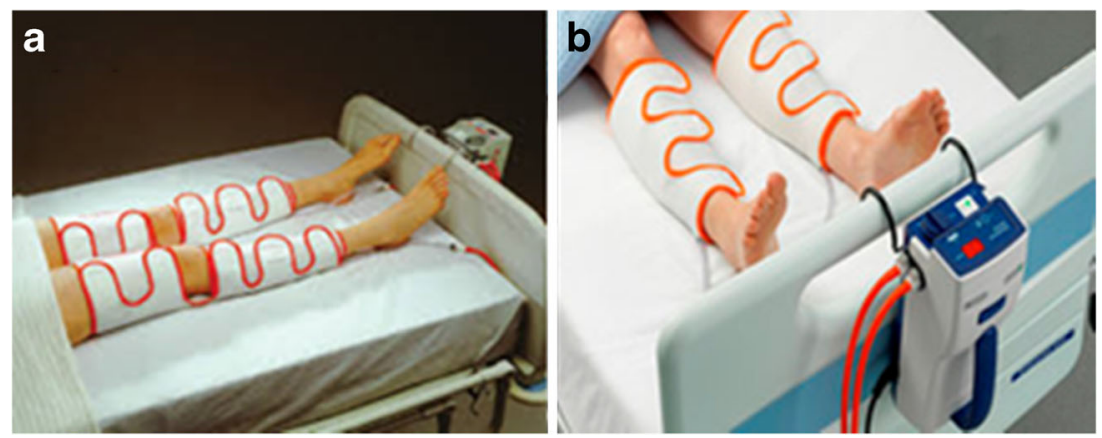
effects seen by calf devices [22]. This increased pressure requirement is often the cause of increased pain and discomfort during application of venous foot pumps and poorer patient compliance.

Hemodynamic comparison of foot compression to calf compression shows foot pumps to be inferior with less peak velocity, duration of effect, and expelled venous volume [23]. The inferior venous flow dynamics of foot pumps however, do not always correlate with clinical outcomes. Despite the inferiority in venous flow dynamics, a clinical study of 184 highrisk trauma patients showed venous foot pumps as effective as thigh IPC devices. Retrospective analysis revealed no statistical difference in rate of DVT for patients utilizing a thigh IPC device, $7 \%$, versus the $3 \%$ in those using a foot pump [24]. A more recent prospective randomized study of 372 trauma patients had conflicting results, however. Foot pumps were found to be inferior to other prevention modalities as DVT rates were $5.7 \%$ in patients using foot pumps, $2.5 \%$ with use of bilateral sequential compression devices and stockings, and $0.8 \%$ for the group of patients provided low-molecularweight heparin [25].

\section{Neuromuscular Electrical Stimulation}

Muscle contraction remains one of the easiest ways to decrease stasis and increase venous return. Upwards of $80 \%$ of lower extremity venous return is attributed to muscle contraction. Comatose or neurologically injured trauma patients, however, are unable to contract their muscles to prevent venous stasis. In this sub-group of patients with contraindications to pharmacologic prophylaxis and/or unavailability of other mechanical prophylaxis methods, neuromuscular electrical stimulation devices may be another option for VTE prevention.

In a study of 40 healthy subjects, electrical foot stimulation was non-inferior relative to standard intermittent pneumatic compression with regard to hemodynamic assessment of venous blood flow in the lower extremity. Most interesting perhaps, were the responses to a questionnaire in which a majority of participants indicated that both treatments were uncomfortable, $92.5 \%$ for intermittent pneumatic compression and $82.5 \%$ for electrical foot stimulation. A majority of the participants $(62.5 \%)$ found the intermittent pneumatic compression device treatment to be more comfortable than the electrical foot stimulation treatment [26].

\section{Synergy of Prophylaxis}

Just as Virchow's model of thrombosis is multifactorial, VTE prevention may be best managed in a multimodal approach. When it comes to current practices in VTE prevention, applying two different modes of prophylaxis is frequently recommended for the high-risk patients. However, the data to support this practice, i.e., are the two methods additive in their prevention of VTE, is sparse.

Adding GCS to legs already being treated with IPC devices was reviewed in a physiologic study of participants with duplex imaging and did not produce any further augmentation of peak venous velocity [27]. With regard to actual clinical outcomes, the addition of GCS to a post-operative regimen of IPC devices similarly did not show a synergistic effect or decrease in DVT rates of the lower extremity [28]. Similarly, no difference was seen when studying orthopedic surgery patients and the rate of DVT after total knee or hip replacement with the addition of GCS to the use of venous foot pumps (2.7 versus $2.3 \%, p=0.07)$. Patient compliance also seems to be improved with the use of IPC devices and omission of GCS [29].

The addition of pharmacologic prophylaxis to mechanical methods of prophylaxis does seem beneficial, however. In a meta-analysis of 17 studies which included 4673 randomized surgical patients wearing GCS or IPC devices, the addition of pharmacologic prophylaxis to mechanical prophylaxis after surgery further decreased the risk of DVT by $44 \%$ (RR 0.56 , $95 \%$ CI $0.45-0.69, p \leq 0.001$ ) [30•]. As part of safety analysis, 15 of these studies tracked bleeding complications and found a small increase in minor or major bleeding events (RR 1.74, $95 \%$ CI 1.29-2.34, $p<0.001)$. These results are similar to findings of a larger meta-analysis that included 70 studies and a mixed population of 16,164 hospitalized patients. Adding pharmacological prophylaxis to the use of intermittent pneumatic compression devices reduced the risk for DVT by $46 \%$ (RR 0.54; $95 \%$ CI 0.32-0.91; $p=0.02$ ) compared with intermittent pneumatic compression devices alone [5].

These, among other studies, have led to the current CHEST guideline recommendations for major trauma patients at high risk for VTE (including those with acute spinal cord injury, traumatic brain injury, and spinal surgery for trauma) to have mechanical prophylaxis added to pharmacologic prophylaxis when not anatomically contraindicated by lower-extremity injury. However, this practice is only a Grade 2C "suggestion" [4].

\section{DVT Risk Reduction}

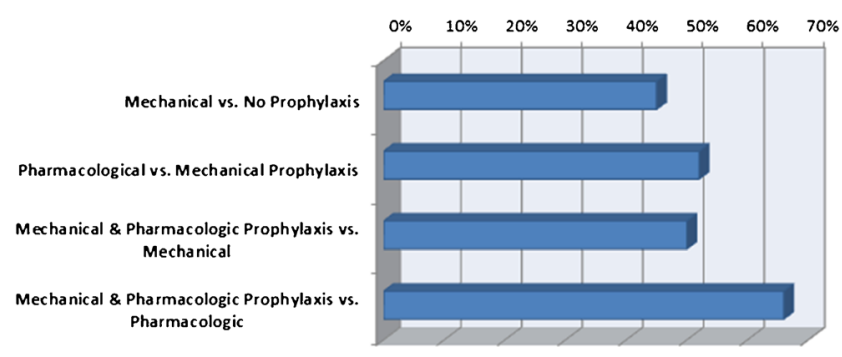

Fig. 3 Summary of DVT risk reduction percentages based on metaanalysis of VTE prevention practices in trauma patients performed by Barrera et al. [18••] 


\section{Contraindications and Pitfalls}

There are very few contraindications to mechanical VTE prophylaxis and overall their safety has led to widespread recommendation for their use $[4 \cdot, 31]$. Adverse complications of mechanical prophylaxis are rare but there have been documented cases of skin necrosis, falls, compartment syndrome, peroneal nerve palsy, and pulmonary embolism [32-34].

Congestive heart failure has been cited as a contraindication to the use of mechanical prophylaxis as the increased venous return could be poorly tolerated and facilitate cardiac failure. The exact physiology of this was disproved by Ringley and colleagues who studied the use of pneumatic compression devices on the lower extremities of patients with pulmonary artery catheters. There was no significant change in any hemodynamic parameter measured and actually a trend toward a decrease in mean arterial pressure, pulmonary artery wedge pressure, and systemic vasculature resistance [33].

Another commonly cited contraindication to the use of lower-extremity mechanical prophylaxis is the presence of a lower-extremity DVT. Although listed as a contraindication in most manufacturer inserts, this is also largely theoretical, as only one case report of pulmonary embolism incited by application of pneumatic compression boots has been reported [34]. In contrast, current guidelines by the American College of Chest Physicians recommend ambulation and the use of elastic compression stockings in patients with DVT. The Ninth ACCP Conference on Antithrombotic and Thrombolytic Therapy observed that post-thrombotic syndrome occurs in upwards of $50 \%$ of patients diagnosed with acute symptomatic DVT and assigned a grade $2 \mathrm{~B}$ recommendation for the use of graduated elastic compression stockings for 2 years after the onset of proximal DVT and anticoagulation treatment [35].

Theoretically, compression devices with circumferential compression decrease the cross-sectional area of the extremity, inhibit surface tension of fascial planes, and impair oxygenation to predispose the patient to compartment syndrome [36]. In the past 30 years, very few cases of compartment syndrome attributable to intermittent pneumatic compression devices have been reported and nearly all have been linked to lithotomy position. Peroneal palsy has also been reported in four cases [37] and is easily avoided by proper application of knee-high compression devices below the fibular head.

\section{Conclusions}

Mechanical prophylaxis has an important part of VTE prophylaxis in trauma patients who often concomitantly have both high VTE and high bleeding risk. Within the confines of studies, of which compression devices are generally required to be worn by the patient at least $75 \%$ of the time, both GCS and IPC reduce the occurrence of DVT by over $40 \%$ compared to no prophylaxis at all. The efficacy of GCS and IPC devices for mechanical VTE prophylaxis are similar. While these garments and devices may come in various shapes and sizes, the literature to date has shown no clinically significant difference in VTE outcomes when it comes to thigh versus knee length, or sequential versus graded compression. Due to poor compliance and increased risk of skin complications, current practice guidelines recommend IPC over GCS as the primary means of mechanical prophylaxis. An observational study of 87,107 pairs of patients matched for propensity to receive VTE prophylaxis found that those managed with pharmacologic VTE prophylaxis had significantly lower risk of death (ICU subhazard ratio $0.82,95 \%$ CI $0.78-0.85$; hospital subhazard ratio $0.82,95 \%$ CI $0.79-0.85 ; p=0.001$ ) compared to those receiving only mechanical VTE prophylaxis [38•]. The risk reduction of VTE events with mechanical prophylaxis, while substantial, is likely not enough in patients who are at a very high risk of VTE (Fig. 3). Therefore, pharmacologic prophylaxis should be employed as soon as possible.

\section{Compliance with Ethical Standards}

Conflict of Interest Drs. Weinberger and Cipolle declare no conflicts of interest.

Human and Animal Rights and Informed Consent This article does not contain any studies with human or animal subjects performed by either of the authors.

\section{References}

Papers of particular interest, published recently, have been highlighted as:

- Of importance

-• Of major importance

1. Virchow RLK. Die Verstopfung den Lungenarterie und ihre Flogen. Beitr Exper Path Physiol. 1846;2:1.

2. Branco BC et al. Thromboelastogram evaluation of the impact of hypercoagulability in trauma patients. Shock. 2014;41(3):200-7.

3. Geerts WH et al. A prospective study of venous thromboembolism after major trauma. N Engl J Med. 1994;331(24):1601-6.

4. Gould MK, Garcia DA, Wren SM, Karanicolas PJ, Arcelus J, Heit JA, Samama CM; American College of Chest Physicians. Prevention of VTE in nonorthopedic surgical patients: Antithrombotic Therapy and Prevention of Thrombosis, 9th ed: American College of Chest Physicians Evidence-Based Clinical Practice Guidelines. Chest 2012;141:e227-277. Includes evidence based guidelines pertaining to VTE prophylaxis in trauma patients.

5. Ho KM, Tan AJ. "Stratified meta-analysis of intermittent pneumatic compression to the lower limbs to prevent venous thromboembolism in hospitalized patients." Circulation (2013);128(9):1003-20. doi:10.1161/CIRCULATIONAHA.113.002690. Large metaanalysis comparing the efficacy of pneumatic compression boots to various other VTE prophylaxis strategies. Patient population is largely surgical. 
6. Chen AH et al. Intermittent pneumatic compression devices - physiological mechanisms of action. Eur J Vasc Endovasc Surg. 2001;21(5):383-92.

7. Comerota AJ, Chouhan V, Harada RN, et al. The fibrinolytic effects of intermittent pneumatic compression: mechanism of enhanced fibrinolysis. Ann Surg. 1997;226:306-13.

8. Hill J, Treasure T. Reducing the risk of venous thromboembolism (deep vein thrombosis and pulmonary embolism) in inpatients having surgery: summary of NICE guidance. BMJ. 2007;334(7602): 1053-4.

9. Lawrence D, Kakkar VV. Graduated, static, external compression of the lower limb: a physiological assessment. Br J Surg. 1980;67(2):119-21.

10. Sachdeva A, Dalton M, Amaragiri SV, Lees T. Graduated compression stockings for prevention of deep vein thrombosis. Cochrane Database Syst Rev. 2014;12:CD001484. doi:10.1002/14651858.CD001484. pub3. The most recent review of graduated compression stockings and their impact on DVT and PE outcomes.

11. Kierkegaard A, Norgren L. Graduated compression stockings in the prevention of deep vein thrombosis in patients with acute myocardial infarction. Eur Heart J. 1993;14:1365-8.

12. Sajid, Muhammad S., et al. "Knee length versus thigh length graduated compression stockings for prevention of deep vein thrombosis in postoperative surgical patients." The Cochrane Library (2012).

13. Heath DI, Kent SJ, Johns DL, Young TW. Arterial thrombosis associated with graduated pressure antiembolic stockings. Br Med J (Clin Res Ed). 1987;295:580.

14. Engineered compression sleeves. Vascular Studies Unit at South Manchester University Hospital http://www.ntu.ac.uk/apps/ research/groups/5/home.aspx/project/143873/overview/ compression_garments.

15. Eppsteiner RW et al. Mechanical compression versus subcutaneous heparin therapy in postoperative and posttrauma patients: a systematic review and meta-analysis. World J Surg. 2010;34(1):10-9.

16. Fedullo P, Brewer R, Rogers K, et al. Comparison of thigh and calf length intermittent pneumatic compression devices for venous thrombosis in trauma patients. Boston: Presented at the annual meeting of the American Thoracic Society; 1994.

17. Proctor $\mathrm{MC}$ et al. A clinical comparison of pneumatic compression devices: the basis for selection. J Vasc Surg. 2001;34(3):459-64.

18.• Barrera, Luis M., et al. "Thromboprophylaxis for trauma patients." The Cochrane Library (2013). A comprehensive review of mechanical and pharmacologic thromboprophylaxis specific to trauma patients.

19. Kakkos SK et al. Comparison of two intermittent pneumatic compression systems: a hemodynamic study. Int Angiol. 2005;24(4):330.

20. Salzman EW et al. Effect of optimization of hemodynamics on fibrinolytic activity and antithrombotic efficacy of external pneumatic calf compression. Ann Surg. 1987;206(5):636.

21. Knight MTN, Dawson R. Effect of intermittent compression of the arms on deep venous thrombosis in the legs. Lancet. 1976;308(7998):1265-8.

22. Whitelaw GP et al. Evaluation of intermittent pneumatic compression devices. Orthopedics. 2001;24(3):257.

23. Delis KT et al. Enhancing venous outflow in the lower limb with intermittent pneumatic compression. A comparative hemodynamic analysis on the effect of foot vs. calf vs. foot and calf compression. Eur J Vasc Endovasc Surg. 2000;19(3):250-60.

24. Spain DA et al. Comparison of sequential compression devices and foot pumps for prophylaxis of deep venous thrombosis in high-risk trauma patients/discussion. Am Surg. 1998;64(6):522.

25. Knudson $\mathrm{MM}$ et al. Use of low molecular weight heparin in preventing thromboembolism in trauma patients. J Trauma Acute Care Surg. 1996;41(3):446-59.

26. Czyrny JJ et al. Electrical foot stimulation: a potential new method of deep venous thrombosis prophylaxis. Vascular. 2010;18(1):20-7.

27. Keith SL et al. Do graduated compression stockings and pneumatic boots have an additive effect on the peak velocity of venous blood flow? Arch Surg. 1992;127(6):727-30.

28. Limpus A et al. Mechanical thromboprophylaxis in critically ill patients: a systematic review and meta-analysis. Am J Crit Care. 2006;15(4):402-12.

29. Pitto RP, Young S. Foot pumps without graduated compression stockings for prevention of deep-vein thrombosis in total joint replacement: efficacy, safety and patient compliance. A comparative, prospective clinical trial. Int Orthop. 2008;32:331-6.

30. Zareba $\mathrm{P}$ et al. Meta-analysis of randomized trials comparing combined compression and anticoagulation with either modality alone for prevention of venous thromboembolism after surgery. Br J Surg. 2014;101(9):1053-62. Meta-analysis of surgical patients comparing VTE outcomes of mechanical compression as prophylaxis with and without the addition of pharmacologic anticoagulation.

31. Rogers FB, Cipolle MD, Velmahos G, Rozycki G, Luchette FA. Practice management guidelines for the prevention of venous thromboembolism in trauma patients: the EAST practice management guidelines work group. J Trauma. 2002;53(1):142-64.

32. Boelig $\mathrm{MM}$ et al. Are sequential compression devices commonly associated with in-hospital falls? A myth-busters review using the patient safety net database. J Patient Saf. 2011;7(2):77-9.

33. Ringley $\mathrm{CD}$ et al. Evaluation of pulmonary arterial catheter parameters utilizing intermittent pneumatic compression boots in congestive heart failure. Am Surg. 2002;68(3):286-90.

34. Siddiqui AU, Buchman TG, Hotchkiss RS. Pulmonary embolism as a consequence of applying sequential compression device on legs in a patient asymptomatic of deep vein thrombosis. J Am Soc Anesthesiol. 2000;92(3):880-0.

35. Kearon $\mathrm{C}$ et al. Antithrombotic therapy for VTE disease: antithrombotic therapy and prevention of thrombosis: American College of Chest Physicians evidence-based clinical practice guidelines. CHEST J. 2012;141(2 suppl):e419S-94S.

36. Gilbart MK et al. Anterior tibial compartment pressures during intermittent sequential pneumatic compression therapy. Am J Sports Med. 1995;23(6):769-72.

37. Datta I et al. Complications related to deep venous thrombosis prophylaxis in trauma: a systematic review of the literature. J Trauma Manag Outcomes. 2010;4(1):e11.

38. Craig CM et al. Thrombosis prophylaxis and mortality risk among critically ill adults. CHEST J. 2014;146(1):51-7. Large multicenter, observational trial investigating the mortality benefit of VTE prophylaxis. 